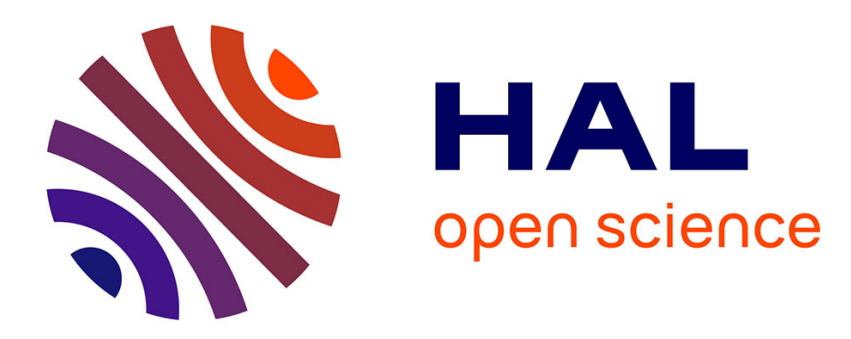

\title{
An example of high order Residual Distribution scheme using non Lagrange elements
}

Remi Abgrall, Jirka Trefilick

\section{To cite this version:}

Remi Abgrall, Jirka Trefilick. An example of high order Residual Distribution scheme using non Lagrange elements. Journal of Scientific Computing, 2009, 45 (1-3), pp.3-25. inria-00403691

\section{HAL Id: inria-00403691 https://hal.inria.fr/inria-00403691}

Submitted on 12 Jul 2009

HAL is a multi-disciplinary open access archive for the deposit and dissemination of scientific research documents, whether they are published or not. The documents may come from teaching and research institutions in France or abroad, or from public or private research centers.
L'archive ouverte pluridisciplinaire HAL, est destinée au dépôt et à la diffusion de documents scientifiques de niveau recherche, publiés ou non, émanant des établissements d'enseignement et de recherche français ou étrangers, des laboratoires publics ou privés. 


\title{
An example of high order Residual Distribution scheme using non Lagrange elements
}

\author{
R. Abgrall ${ }^{(1,2)}$ and J. Trefflick ${ }^{(1,3)}$ \\ (1) INRIA Bordeaux Sud Ouest, 33405 Talence, France \\ (2) Institut Polytecnhique de Bordeaux, 33405 Talence, France \\ (3) CTU Prag,
}

June 8, 2009

\begin{abstract}
We are interested in the numerical approximation of non linear hyperbolic problems. The particular class of schemes we are interested in are the so-called Residual Distribution schemes. In their current form, they rely on the Lagrange interpolation of the point values of the approximated functions. This interpretation of the degrees of freedom as point values plays a fundamental role in the derivation of the schemes. The purpose of the present paper is to show that some non Lagrange elements can also do the job, and maybe better. This opens the door to isogeometric analysis in the framework of RDS schemes.
\end{abstract}

We are interested in the numerical approximation of linear and non linear hyperbolic problems. The particular class of schemes we are interested in are the so-called Residual Distribution schemes. They can be traced back to the early work of P.L. Roe [1] and Ni [2], but also to the stabilized finite element schemes such as the Hughes' SUPG scheme $[3,4,5]$. Their main characteristics are the following: (i) they have a natural formulation on unstructured meshes, (ii) their stencil is the most possible compact one to reach a given order of accuracy, (iii) their parallelization is straightforward. These three properties are shared in common with the Discontinuous Galerkin scheme, but here, thanks to the conformal nature of the approximation, the number of degrees of freedom is reduced by a large factor, as this can be seen on table 1 .

\begin{tabular}{|c|cc||cc|}
\hline & \multicolumn{2}{|c|}{$2 \mathrm{D}$} & \multicolumn{2}{c|}{$3 \mathrm{D}$} \\
\hline Order & DG & RDS & DG & RD \\
2 & $6 n_{s}$ & $n_{s}$ & $24 n_{s}$ & $n_{s}$ \\
3 & $12 n_{s}$ & $4 n_{s}$ & $40 n_{s}$ & $8 n_{s}$ \\
4 & $20 n_{s}$ & $9 n_{s}$ & $80 n_{s}$ & $27 n_{s}$ \\
\hline
\end{tabular}

Table 1: Number of degrees of freedom for third and fourth order approximation in the case of a triangular/tet mesh. DG stands for Discontinuous Galerkin, RD for Residual Distribution.

In previous papers, we, and others $[6,7,8,9,10,11,12,13]$, have shown how to combine monotonicity preserving properties and very high accuracy $(\geq 2)$ on general conformal meshes, or non conformal meshes $[14,15]$. One of the key ingredient in the construction is that the degrees of freedom can be interpreted as point values. The purpose of the present paper is to show that some non Lagrange elements can also do the job. This opens the door to isogeometric analysis [16] in the framework of RDS schemes.

The format of the paper is as follows. In a first part, we recall what are these Residual Distribution schemes, and show the construction of high order schemes. A monotonicity principle, or variation diminishing one, plays a key role. In the second part, we provide examples for scalar steady non linear hyperbolic equations. The third part discuss the extension to the unsteady case for a wave model. Conclusion follows. 


\section{The residual distribution schemes}

\subsection{Introduction}

Let us consider the following scalar model equation,

$$
\begin{array}{ll}
\operatorname{div} f(u)=S(x) & x \in \Omega \subset \mathbb{R}^{d} \\
u=g & \text { weakly on the inflow boundary } \Gamma_{-}
\end{array}
$$

where $\Gamma_{-}=\left\{x \in \partial \Omega, \nabla_{u} f(u) \cdot \vec{n}(x)<0\right\}, \vec{n}(x)$ is the outward unit normal of $\Omega$ at $x$. In (1), $u$ and $g$ belong to $\mathbb{R}$, and the flux $f$ has $d$ components, namely $f=\left(f_{1}, \ldots, f_{d}\right)$. We assume that $f$ is $C^{1}$ and $g$ belongs to $L^{\infty}(\Gamma)$. The discussion will be developed using that scalar model, with $d=2$, however extensions to systems and the case $d=3$ are rather straightforward.

We consider a triangulation of $\Omega$ denoted by $\mathcal{T}_{h}$. The triangles are $\left\{T_{j}\right\}_{j=1, \ldots, n_{e}}$. We denote by $\Omega_{h}=$ $\cup_{j=1, \ldots, n_{e}} T_{j}$. The vertices of the mesh are denoted by $\left\{M_{i}\right\}_{i=1, n_{v}}$. Besides the usual regularity assumptions we need, we also make the standard assumption that if an element $T$ has a part of an edge on $\Gamma_{h}:=\partial \Omega_{h}$, this full edge is included in $\Omega_{h}$.

In each element $T$, we need an approximation of the solution, say $u^{h}$, and we assume the following form

$$
u_{\mid T}^{h}=\sum_{\sigma_{\ell} \in T} u_{\sigma_{\ell}}\left(\psi_{\sigma_{\ell}}\right)_{\mid T}
$$

In (2), the sum is indexed by degrees of freedom that are seen as points in $T$. A typical example is a Lagrange interpolant. We will assume that the function $u^{h}$ is continuous across edges, i.e. the $\psi_{\sigma_{\ell}}$ are continuous across the edges of $\mathcal{T}_{h}$, so that we write

$$
u^{h}=\sum_{\sigma_{\ell}} u_{\sigma_{\ell}} \psi_{\sigma_{\ell}}
$$

More precisely, given $k \in \mathbb{N}$, we assume that for any function smooth enough $u \in C^{k+1}(\Omega)$, we can define $u^{h}=\pi_{h}(u)$ of this type, such that if $u$ is a polynomial of degree $k$, we have $u=u^{h}$. Then, standard approximation results, se for example [17], show that in $L^{p}$ norms, we have $\left\|u-\pi_{h}(u)\right\| \leq C(u) h^{k+1}$. These properties are true for example using Lagrange polynomials, Bezier, spline representations or NURBS $[18,19]$. We assume that degrees of freedom also live on the boundary of $T$, this is true for any of these examples. Note that this assumption is consistent with the continuity assumption.

Thanks to this, we define, in each element $T$, the total residual $\Phi_{T}$ as

$$
\Phi_{T}=\int_{\partial T} f^{h}\left(u^{h}\right) \cdot \vec{n} d l-\int_{T} S(x) d x
$$

where $f^{h}$ is some approximation of the flux $f$. We precise the assumptions on $f^{h}$ a bit latter in the text. Once this has been done, we consider split-residuals, $\Phi_{\sigma}^{T}$, for $\sigma \in T$, so that they satisfy the conservation property:

$$
\sum_{\sigma \in T} \Phi_{\sigma}^{T}=\Phi_{T}
$$

In order to handle boundary conditions, we need to consider boundary residuals. Let $\Gamma$ be an edge of some triangle $T$ which is on $\Gamma_{h}$, we consider the boundary residual

$$
\Phi_{\Gamma}=\int_{\Gamma}\left(\mathcal{F}\left(u^{h}, u_{-}, \vec{n}\right)-g(x) \cdot \vec{n}\right) d l
$$

where $\left(\mathcal{F}\left(u^{h}, u_{-}, \vec{n}\right)\right.$ is a numerical upwind flux that depends on the trace of $u^{h}$ on $\Gamma$, the boundary condition $u_{-}=g$, with the understanding that the numerical flux vanishes on the non upwind parts of the boundary. Then, we consider split-residuals $\Phi_{\sigma}^{\Gamma}$, for $\sigma \in \Gamma$, so that they satisfy the conservation property:

$$
\sum_{\sigma \in \Gamma} \Phi_{\sigma}^{\Gamma}=\Phi_{\Gamma}
$$


Once this has been done, the scheme writes : find $u^{h}$ such that for any degree of freedom $\sigma$,

- If $\sigma \notin \partial \Omega_{h}$,

$$
\Sigma\left(u^{h}\right):=\sum_{T \ni \sigma} \Phi_{\sigma}^{T}=0
$$

- If $\sigma \in \partial \Omega_{h}$

$$
\Sigma\left(u^{h}\right):=\sum_{T \ni \sigma} \Phi_{\sigma}^{T}+\sum_{\Gamma \subset \partial \Omega^{-}, \Gamma \ni \sigma} \Phi_{\sigma}^{\Gamma}=0 .
$$

We can summarize (7a) and (7b) by

$$
\Sigma\left(u^{h}\right)=\sum_{E \ni i} \Phi_{\sigma}^{T}=0
$$

where $E$ stands either for any triangle $T$ or edge $\Gamma$ that shares $\sigma$.

\subsection{Design principles}

\subsubsection{Consistency with (1)}

What are the design principles on the scheme (7) with (4) so that we have a convergent scheme? The answer to this problem has been provided in [13], and we reproduce the result.

Proposition 1.1. Assume that the mesh is regular, that the flux approximation $f^{h}\left(u^{h}\right)$ is continuous across edges and defines a convergent approximation (in $L^{1}$ of the $C^{1}$ flux $f$. Assume that the residuals satisfy the conservation relations (4) and (6). Assume that the scheme (7) defines a unique $u^{h}$ such that

1. there exist a constant $C(g)$ independent of $h$ such that $\left\|u^{h}\right\|_{L^{2}} \leq C(g)$,

2. there exists $v \in L^{2}(\Omega)$ such that a subsequence of $u^{h}$ converges to $v$ in $L^{2}$,

then $v$ is a weak solution of (1)

The result of [13] was about a first order in time approximation of

$$
\frac{\partial u}{\partial t}+\operatorname{div} f(u)=0
$$

with initial condition. The adaptation to the steady case (1) with boundary conditions and source term is straightforward, and uses exactly the same arguments.

\subsubsection{Accuracy}

Again, we recall previous results, see [13] The key remark is to see that if one can solve (7) accurately, the scheme is formally $r$ order accurate if the split-residual satisfy

$$
\Phi_{\sigma}^{T}=O\left(h^{r+d}\right), \quad \Phi_{\sigma}^{\Gamma}=O\left(h^{k+d-1}\right) .
$$

The reason follows from a simple error analysis. If $\varphi$ is a compactly supported test function, let us denote $\varphi^{h}$ its Lagrange interpolation defined by $\varphi^{h}(\sigma)=\varphi(\sigma)$. Saying that, we assume that within each triangle, the set of degrees of freedom is unisolvant. The examples of section 2 will make that point clearer. Then we 
multiply the relations (7) by $\varphi_{\sigma}^{h}$ and add, then using the conservation relations we obtain

$$
\begin{aligned}
\mathcal{E}\left(u^{h}, \varphi_{h}\right)= & \sum_{\sigma \in \Omega} \varphi(\sigma)\left(\sum_{T \ni \sigma} \Phi_{\sigma}^{T}+\sum_{\Gamma \subset \partial \Omega^{-}, \Gamma \ni \sigma} \Phi_{\sigma}^{\Gamma}\right) \\
= & \int_{\Omega}\left(\operatorname{div} f^{h}\left(u^{h}\right)-S^{h}\left(u^{h}\right)\right) \varphi_{h}(x) d x+\sum_{T \subset \Omega} \frac{1}{\#\{\sigma \in T\}} \sum_{\sigma, \sigma^{\prime} \in T}\left(\varphi(\sigma)-\varphi\left(\sigma^{\prime}\right)\right)\left(\Phi_{\sigma}^{T}-\Phi_{\sigma}^{T, c}\right) \\
+ & \int_{\partial \Omega}\left(\mathcal{F}\left(u^{h}, u_{-}, \vec{n}\right)-f^{h}\left(u^{h}\right) \cdot \vec{n}\right) \varphi_{h}(x) d l+\sum_{\Gamma \subset \partial \Omega} \frac{1}{\#\{\sigma \in \Gamma\}} \sum_{\sigma, \sigma^{\prime} \in \Gamma}\left(\varphi(\sigma)-\varphi\left(\sigma^{\prime}\right)\right)\left(\Phi_{\sigma}^{\Gamma}-\Phi_{\sigma}^{\Gamma, c}\right) \\
= & -\int_{\Omega} \nabla \varphi_{h}(x) \cdot f^{h}\left(u^{h}\right)+\int_{\partial \Omega} \varphi_{h}(x) f^{h}\left(u^{h}\right) \cdot \vec{n} d l+\int_{\Omega} \varphi_{h}(x) S^{h}\left(u^{h}\right) d x \\
& \quad+\int_{\partial \Omega}\left(\mathcal{F}\left(u^{h}, u_{-}, \vec{n}\right)-f^{h}\left(u^{h}\right) \cdot \vec{n}\right) \varphi_{h}(x) d l \\
& \quad+\sum_{T \subset \Omega} \frac{1}{\#\{\sigma \in T\}} \sum_{\sigma, \sigma^{\prime} \in T}\left(\varphi(\sigma)-\varphi\left(\sigma^{\prime}\right)\right)\left(\Phi_{\sigma}^{T}-\Phi_{\sigma}^{T, c}\right) \\
& \quad+\sum_{\Gamma \subset \partial \Omega} \frac{1}{\#\{\sigma \in \Gamma\}} \sum_{\sigma, \sigma^{\prime} \in \Gamma}\left(\varphi(\sigma)-\varphi\left(\sigma^{\prime}\right)\right)\left(\Phi_{\sigma}^{\Gamma}-\Phi_{\sigma}^{\Gamma, c}\right) .
\end{aligned}
$$

where $\varphi_{h}=\pi_{h}(\varphi)$,

$$
\Phi_{\sigma}^{T, c}=\int_{T} \psi_{\sigma}\left(\operatorname{div} f\left(u^{h}\right)-S\left(u^{h}\right)\right) d x, \quad \Phi_{\sigma}^{\Gamma, c}=\int_{\Gamma} \psi_{\sigma}\left(\mathcal{F}\left(u^{h}, u_{-}, \vec{n}\right)-f\left(u^{h}\right) \cdot \vec{n}\right) d x
$$

and $\psi_{\sigma} \in \mathbb{P}^{k}(T)$ such that $\psi_{\sigma}\left(\sigma^{\prime}\right)=\delta_{\sigma^{\prime}}^{\sigma}$.

Following again [13], have the following result:

Proposition 1.2. If the solution $u$ is smooth enough and the residual, applied to the $\mathbb{P}_{k}$ interpolant of $u$ satisfy

$$
\Phi_{\sigma}^{T}\left(u^{h}\right)=\mathcal{O}\left(h^{k+d}\right)
$$

and

$$
\Phi_{\sigma}^{\Gamma}=\mathcal{O}\left(h^{k+d-1}\right),
$$

if moreover the approximation $f^{h}\left(u^{h}\right)$ is $k+1$-order accurate, then the truncation error satisfies

$$
\left|\mathcal{E}\left(u^{h}, \varphi^{h}\right)\right| \leq C(\varphi, f, u) h^{k+1} .
$$

The constant $C(\varphi, u)$ depends only on $\varphi$ and $u$.

We start by a lemma

Lemma 1.3. For the steady problem (1), if the solution $u$ is smooth, we have

$$
\int_{\partial T} f^{h}\left(u^{h}\right) \cdot \vec{n} d l-\int_{T} S(x) d x=O\left(h^{k+d}\right)
$$

and

$$
\int_{\partial T}\left(\mathcal{F}\left(u^{h}, u_{-}, \vec{n}\right)-f^{h}\left(u^{h}\right) \cdot \vec{n}\right) d l=O\left(h^{k+d-1}\right)
$$

provided that the approximation $f^{h}\left(u^{h}\right)$ is $k+1$ th order accurate and the numerical flux $\mathcal{F}$ is Lipschitz continuous. 
Proof. We have, using the fact that (1) is a steady problem,

$$
\begin{aligned}
\int_{\partial T} f^{h}\left(u^{h}\right) \cdot \vec{n} d l-\int_{T} S(x) d x & =\int_{\partial T}\left(f^{h}\left(u^{h}\right) \cdot \vec{n}-f(u)\right) d l \\
& =O\left(h^{k+1}\right) \times|\partial T|=O\left(h^{k+d}\right) .
\end{aligned}
$$

On the boundary, we have

$$
\begin{aligned}
\int_{\partial T}\left(\mathcal{F}\left(u^{h}, u_{-}, \vec{n}\right)-g(x) \cdot \vec{n}\right) d l & =\int_{\partial T}\left(\mathcal{F}\left(u^{h}, u_{-}, \vec{n}\right)-g(x) \cdot \vec{n}\right) d l+\int_{\partial T}\left(\mathcal{F}\left(u, u_{-}, \vec{n}\right)-g(x) \cdot \vec{n}\right) d l \\
& =\int_{\partial T}\left(\mathcal{F}\left(u^{h}, u_{-}, \vec{n}\right)-\mathcal{F}\left(u, u_{-}, \vec{n}\right)\right) d l
\end{aligned}
$$

and the result follows because of the approximation inequality and since the numerical flux is Lipschitz continuous.

Proof of proposition 1.2. This inequality is a consequence of (8) because we have

$$
\begin{aligned}
-\int_{\Omega} \nabla \varphi_{h}(x) \cdot f^{h}\left(u^{h}\right) & +\int_{\partial \Omega} \varphi_{h}(x) f^{h}\left(u^{h}\right) \cdot \vec{n} d l+\int_{\Omega} \varphi^{h}(x) S^{h}\left(u^{h}\right) d x= \\
& \left(-\int_{\Omega} \nabla \varphi_{h}(x) \cdot f(u)+\int_{\partial \Omega} \varphi_{h}(x) f(u) \cdot \vec{n} d l+\int_{\Omega} \varphi^{h}(x) S^{h}(u) d x\right) \\
& +\left(-\int_{\Omega} \nabla \varphi_{h}(x) \cdot\left(f(u)-f^{h}\left(u^{h}\right)\right)\right. \\
& \left.+\int_{\partial \Omega} \varphi_{h}(x)\left(f(u)-f^{h}\left(u^{h}\right)\right) \cdot \vec{n} d l+\int_{\Omega} \varphi^{h}(x)\left(S^{h}(u)-S^{h}\left(u^{h}\right)\right) d x\right)
\end{aligned}
$$

where $u^{h}=\pi_{h}(u)$. From standard interpolation results [17], we have $\left|\varphi^{h}\right| \leq C$ and $\left|\nabla \varphi^{h}\right| \leq C^{\prime}, \mid f^{h}\left(u^{h}\right)-$ $f(u) \mid \leq C(u, f) h^{k+1}$ and $\left|S^{h}\left(u^{h}\right)-S(u)\right| \leq C(u, S) h^{k+1}$. so that (10) is in norm smaller that $C(u, f, S) h^{k+1}$ for a suitable constant $C(u, f, S)$.

From lemma 1.3, for any $T$ and $\Gamma,\left|\Phi_{\sigma}^{T, c}\right| \leq C(u, f, S) h^{k+d}$ and $\left|\Phi_{\sigma}^{\Gamma, c}\right| \leq C(u, f, S) h^{k+d-1}$ where $d$ is the space dimension.

Then, For any $T$,

$$
\begin{aligned}
\left|\sum_{\sigma, \sigma^{\prime} \in T}\left(\varphi(\sigma)-\varphi\left(\sigma^{\prime}\right)\right)\left(\Phi_{\sigma}^{T}-\Phi_{\sigma}^{T, c}\right)\right| & \leq \sum_{\sigma, \sigma^{\prime} \in T}\left(\left|\varphi(\sigma)-\varphi\left(\sigma^{\prime}\right)\right|\right)\left(\left|\Phi_{\sigma}^{T}\right|+\left|\Phi_{\sigma}^{T, c}\right|\right) \mid \\
& \leq \# \text { of elements } \times N \times\|\nabla \varphi\|_{\infty} h \times C(\varphi, f, S) h^{k+d}
\end{aligned}
$$

where $N$ is the number of degree of freedom in each element. In a regular mesh for a bounded domain, the number of elements sizes like $h^{-d}$ so that in the end, we can find a constant (again denoted by $C$ ) which depends on $u, f, S$ and $\Omega$ such that

$$
\left|\sum_{\sigma, \sigma^{\prime} \in T}\left(\varphi(\sigma)-\varphi\left(\sigma^{\prime}\right)\right)\left(\Phi_{\sigma}^{T}-\Phi_{\sigma}^{T, c}\right)\right| \leq C(u, f, S, \Omega) h^{k+1} .
$$

The last estimation is to be done for the boundary terms. Using the consistency of the numerical flux, we first have

$$
\begin{aligned}
& \left|\int_{\partial \Omega}\left(\mathcal{F}\left(u^{h}, u_{-}, \vec{n}\right)-f^{h}\left(u^{h}, \vec{n}\right)\right) \varphi_{h}(x) d l\right| \leq \int_{\partial \Omega}\left(\left|\mathcal{F}\left(u^{h}, u_{-}, \vec{n}\right)-\mathcal{F}\left(u^{h}, u^{h}, \vec{n}\right)\right|\right) \varphi_{h}(x) d l \\
& \quad \leq L \int_{\partial \Omega}\left|u^{h}-u_{-}\right| \leq C(u, f, \partial \Omega) h^{k+1}
\end{aligned}
$$


Similarly, we have, for any boundary edge, $\left|\Phi_{\sigma}^{\Gamma, c}\right| \leq C(u, f) h^{k+d}$. If the boundary of $\Omega$ is regular, the number of boundary faces is of the order of $h^{-(d-1)}$.

Thus, we get, using again the same arguments,

$$
\left|\sum_{\Gamma \subset \partial \Omega} \sum_{\sigma, \sigma^{\prime} \in \Gamma}\left(\varphi(\sigma)-\varphi\left(\sigma^{\prime}\right)\right)\left(\Phi_{\sigma}^{\Gamma}-\Phi_{\sigma}^{\Gamma, c}\right)\right| \leq C(u, f, \partial \Omega) h^{-d+1} h^{k+d}=C(u, f, \partial \Omega) h^{k+1} .
$$

This completes the proof.

Let us conclude this paragraph by two important remarks.

Remark 1.4. We see that the proof uses two key elements:

- The problem (1) is steady,

- One is able to compute $u^{h}$. This is done in practice via an iterative algorithm because the system (7) is in general non linear. In all the numerical examples, we will consider a simple Jacobi-like iteration,

$$
u_{\sigma}^{k+1}=u_{\sigma}^{k}-\omega_{\sigma}^{k} \Sigma\left(\left(u^{h}\right)^{k}\right)
$$

where $\omega_{\sigma}^{k}$ is a relaxation parameter that can be thought as the ratio of a time step (constraint by a CFL condition) and an area. The sequence $\left(u^{h}\right)^{k}$ is initialized to some value (say $\left.u^{h}=0\right)$ and marched up to convergence. The convergence issue of the sequence is a subtle one, as it will be seen.

The accuracy result will be true, in practice, provided that one is able to construct a convergent sequence $\left(\left(u^{h}\right)^{k}\right)_{k \in \mathbb{N}}$, that is, for any $\varepsilon>0$, one can find $N_{\varepsilon}$ such that

$$
n \geq N_{\varepsilon}, \text { then }\left|\Sigma\left(\left(u^{h}\right)^{k}\right)\right| \leq \varepsilon .
$$

The algorithm can be stopped provided that $\varepsilon=O\left(h^{k}\right)$.

\subsubsection{Monotonicity preservation}

In the previous versions of the RD scheme, the degrees of freedom were Lagrange points, so that $u_{\sigma}^{h}$ is the value of $u^{h}$ at $\sigma$. In that case, the iterative scheme is designed in such a way that for any $k \in \mathbb{N}$,

$$
\max _{\sigma}\left|u_{\sigma}^{k}\right| \leq \max _{\sigma} \max \left(\|g\|_{\infty}, \max _{\sigma}\left|u_{\sigma}^{0}\right|\right)
$$

Indeed, the scheme is designed so that for any $\sigma$,

$$
\max _{\sigma^{\prime} \in V(\sigma)}\left|u_{\sigma}^{k}\right| \leq \max _{\sigma^{\prime} \in V(\sigma)}\left|u_{\sigma}^{k-1}\right|
$$

where $V(\sigma)$ is the set of neighbors of $\sigma, \sigma$ included. Note that in this case, we are not asking for

$$
\left\|\left(u^{h}\right)^{k}\right\| \leq C
$$

since it is well known that the Lagrange interpolation, for degree larger than 2, suffers from the Gibbs phenomena.

Another way of thinking is precisely to try to enforce the constraint (12) globally. Assume that we have a scheme that writes:

$$
\Phi_{\sigma}^{E}=\sum_{\sigma^{\prime} \in T} c_{\sigma \sigma^{\prime}}^{T}\left(u_{\sigma}-u_{\sigma^{\prime}}\right)
$$

where $E$ is either a triangle (case of an internal degree of freedom) or a boundary edge $\Gamma$ (case of a boundary degree of freedom), with

$$
\text { for any } \sigma, \sigma^{\prime}, c_{\sigma \sigma^{\prime}}^{T} \geq 0 \text {. }
$$


Using the iterative scheme (11), it is clear that

$$
\left|u_{\sigma}^{k+1}\right| \leq \max _{\sigma^{\prime} \in V_{\sigma}}\left|u_{\sigma^{\prime}}^{k}\right|
$$

provided that

$$
\omega_{\sigma} \leq\left(\sum_{E \ni \sigma} \sum_{\sigma^{\prime} \in T} c_{\sigma \sigma^{\prime}}\right)^{-1}
$$

where $E$ is either a triangle or a boundary edge.

If the basis functions $\psi_{\sigma}$ are positive we see that

$$
\mid\left(u^{h}\right)^{n+1} \leq \max _{\sigma} \max \left(\|g\|_{\infty}, \max _{\sigma}\left|u_{\sigma}^{0}\right|\right)
$$

An example of such a split-residual is given by the following Lax-Friedrich like residual: we first approximate $f(u)$ by

$$
\begin{gathered}
f^{h}\left(u^{h}\right):=f\left(u^{h}\right) . \\
\Phi_{\sigma}^{T}=\frac{\Phi^{T}}{N_{T}}+\alpha_{T}\left(u_{\sigma}-\bar{u}^{T}\right)
\end{gathered}
$$

with

$$
\bar{u}^{T}=\frac{\sum_{\sigma^{\prime} \in T} u_{\sigma^{\prime}}}{N_{T}}, \quad \alpha_{T} \geq \max _{\sigma^{\prime} \in T} \int_{T} ? ? ? ?
$$

and $N_{T}$ being the number of degrees of freedom in $T$. This family of split residuals defines a scheme that is only first order accurate.

\subsection{Construction of high order schemes}

How can we construct a scheme that is both monotonicity preserving and high order accurate. Using the remark contained in Lemma 1.3, one possibility is to look for real numbers $\beta_{\sigma}^{E}\left(u^{h}\right)$ ( $E$ triangle or boundary edge) such that

$$
\Phi_{\sigma}^{E}=\beta_{\sigma}^{E}\left(u^{h}\right) \Phi_{T},
$$

that are uniformly bounded. This ensure that $\Phi_{\sigma}^{T}=O\left(h^{k+d}\right)$ and $\Phi_{\sigma}^{\Gamma}=O\left(h^{k+d-1}\right)$.

The question is to define the $\beta$ s such that the scheme is both high order accurate and monotonicity preserving.

A first step is the following : using a monotonicity preserving scheme (think of the Lax Friedrichs scheme) which residuals are denoted by $\Phi_{\sigma}^{L, T}$ which satisfies (13), we formally write

$$
\begin{aligned}
\Phi_{\sigma}^{H, T} & =\frac{\Phi_{\sigma}^{H, T}}{\Phi_{\sigma}^{L, T}} \Phi_{\sigma}^{L, T} \\
& =\sum_{\sigma^{\prime} \in T}\left(\frac{\Phi_{\sigma}^{H, T}}{\Phi_{\sigma}^{L, T}}\right) c_{\sigma \sigma^{\prime}}^{L}\left(u \sigma-u_{\sigma^{\prime}}\right) \\
& =\sum_{\sigma^{\prime} \in T} c_{\sigma \sigma^{\prime}}^{H}\left(u \sigma-u_{\sigma^{\prime}}\right)
\end{aligned}
$$

with $c_{\sigma \sigma^{\prime}}^{H}=\left(\frac{\Phi_{\sigma}^{H, T}}{\Phi_{\sigma}^{L}, T}\right) c_{\sigma \sigma^{\prime}}^{L}$. Hence, since $c_{\sigma \sigma^{\prime}}^{L} \geq 0$, we have $c_{\sigma \sigma^{\prime}}^{H} \geq 0$ provided that $\frac{\Phi_{\sigma}^{H, T}}{\Phi_{\sigma}^{L, T}} \geq 0$. Setting

$$
x_{\sigma}=\frac{\Phi_{\sigma}^{L, T}}{\Phi^{T}} \text { and } \beta_{\sigma}=\frac{\Phi_{\sigma}^{H, T}}{\Phi^{T}},
$$


the conservation and monotonicity preserving condition become

$$
\sum_{\sigma \in T} x_{\sigma}=\sum_{\sigma \in T} \beta_{\sigma}=1 \text { and for any } \sigma \in T, x_{\sigma} \beta_{\sigma} \geq 0
$$

The problem is to find a mapping $\left(x_{\sigma}\right)_{\sigma \in T} \mapsto\left(\beta_{\sigma}\right)_{\sigma \in T}$ that satisfies the conditions (19). This mapping cannot be linear according to Godunov's theorem.

An extensive discussion of these relations is done in [13], in particular we provide a geometrical interpretation of these relations. Among the many mappings that satisfy (19), we have chosen

$$
\beta_{\sigma}=\frac{x_{\sigma}^{+}}{\sum_{\sigma^{\prime} \in T} x_{\sigma^{\prime}}^{+}}
$$

which is always well defined because $\sum_{\sigma^{\prime} \in T} x_{\sigma^{\prime}}^{+} \geq 1$.

Unfortunately, as we see in the next section, the resulting scheme (i.e. (7) with (17) and (20) using the Lax Friedrichs scheme) is over compressive. The same problem would occur with other first order spli=residuals, for example those constructed form standard first order flux, see [10] for some examples. The fundamental reason is that the limitation is done according to monotonicity preserving constraints only, in complete ignorance of what is the physics of the problem, i.e. how up-winding has to be triggered into the scheme. Hence, we need to add some dissipation mechanism without destroying the formal accuracy in order to correct that drawback. . One way of doing that is to add to (17) a dissipative term, namely

$$
d_{T}\left(\varphi^{h}, u^{h}\right)=|T| \sum_{x_{\text {quad }}} \omega_{\text {quad }}\left[\left(\nabla_{u} f\left(u^{h}\right) \cdot \nabla \varphi_{\sigma}\right)\left(x_{\text {quad }}\right)\left(\nabla_{u} f\left(u^{h}\right) \cdot \nabla u^{h}-S\right)\left(x_{\text {quad }}\right)\right]
$$

such that the quadratic form

$$
\left(v^{h}, u^{h}\right) \mapsto \sum_{\sigma} v_{\sigma}^{h}\left(\sum_{E \ni \sigma} \Phi_{\sigma}^{E}+\sum_{T} \theta_{T} h_{T} d_{T}\left(\varphi^{h}, u^{h}\right)\right.
$$

is dissipative. Again, $E$ stands for any element or edge that share $\sigma$. In $(21), h_{T}$ is a the radius of the circumscribed circle/sphere, and $\theta_{T}$ is a parameter that is of the order of 0 in discontinuities and 1 elsewhere. In (21), $x_{\text {quad }}$ can be interpreted as quadrature points and $\omega_{\text {quad }}$ as weights. Saying, we interpret (21) as a discrete version of

$$
\int_{T}\left(\nabla_{u} f(u) \varphi_{\sigma}\right) \cdot\left(\nabla_{u} f(u) \nabla u^{h}-S(x)\right) d x .
$$

However, in [20], we have shown that, at least for linear flux $f(u)=\vec{\lambda} u$, is that a necessary condition is that the quadratic form

$$
q_{K}\left(v_{h}\right):=\sum_{x_{\text {quad }}} \omega_{\text {quad }}\left(\vec{\lambda} \cdot \nabla v_{h}\left(x_{\text {quad }}\right)\right)^{2}
$$

is positive definite whenever the polynomial $\lambda \cdot \nabla v^{h}$ is not identically zero. In the case of polynomial interpolation, we need only one quadrature point (and $\omega_{\text {quad }}=1$ ), for quadratic polynomials, we need three non aligned points (in practice the vertices of the element, and we take $\omega_{\text {quad }}==\frac{1}{3}$, and so on. Details can be found in this reference, we will use this technique in the present paper.

There are many possible choices for the parameter $\theta_{T}$. For example, $\theta_{T}$ is a good choice, even in the case of discontinuous solutions where we have experimentally noticed that no (visible) spurious oscillation occur. However, the best choice we have experimented is

$$
\theta_{T}=\max _{\sigma \in T}\left(\max _{T \ni \sigma} \max _{\sigma^{\prime} \in T} \frac{\left|u_{\sigma^{\prime}}-\bar{u}_{T}\right|}{\left|u_{\sigma^{\prime}}\right|+\left|\bar{u}_{T}\right|+\varepsilon}\right)
$$

with $\varepsilon \approx 10^{-10}$. Here, $\bar{u}_{T}=\left(\sum_{\sigma \in T} u_{\sigma}\right) / N$. 


\subsection{Some comments}

Let us conclude by the following remark : in the RD schemes, we add a term that is dissipative. In contrast to other high order schemes, the effect of this term is not to damp the solution but to increase the accuracy. This is because that more than dissipating the solution, the role of this term is to remove spurious modes. There is a very simple example where it can be seen that spurious modes do exist, unless something is done. Consider the PDE on $[0,1]^{2}$,

$$
\begin{aligned}
& \frac{\partial u}{\partial x}=0 \\
& u(0, y)=u_{0}(y) .
\end{aligned}
$$

The solution is $u(x, y)=u_{0}(y)$. Consider a mesh made of quadrangles, the elements are $K_{i j}=\left[x_{i}, x_{i+1}\right] \times$ $\left[y_{j}, y_{j+1}\right]$ with $x_{i}=i / N$ and $y_{j}=j / N, 0 \leq i, j \leq N-1$. We can construct linear preserving schemes, exactly as we have proceed above. Consider the one which is constructed from a $Q^{1}$ interpolation.

If the set the boundary condition to $u_{i, 0}=(-1)^{i}$ we expect the solution to be an approximation of $u_{i, j}=(-1)^{i}$. Since we have an iterative scheme, from (11), we can initialize either by

or

$$
u_{i j}^{0}= \begin{cases}(-1)^{i} & \text { if } j=0 \\ 0 & \text { else }\end{cases}
$$

$$
u_{i j}^{0}=(-1)^{i+j}
$$

or something else. The second initialization is the check-board mode. The first initialization will give some sequence. The second one is stationary because

$$
\int_{K_{i j}} \frac{\partial u^{h}}{\partial x} d x d y=0
$$

for the check-board mode.

This is an example of a RD scheme that cannot converge. This is why we need to add some selection mechanism, and as we show in the next section, the effect of this "damping" is to improve a lot the accuracy or the solution.

\section{Numerical examples}

We consider two type of approximations: Lagrange polynomials and Bézier approximation. In the case of a triangle with vertices $A_{1}, A_{2}, A_{3}$, we denote by $\mathcal{N}_{A_{i}}$ the linear shape functions at these vertices $\left(\mathcal{N}_{A_{i}}\left(A_{j}\right)=\delta_{i}^{j}\right)$. If, for $k_{1}, k_{2}$ and $k_{3}$ integer such that $k_{1}+k_{2}+k_{3}=n, \alpha_{k 1 k 2 k 3}$ the coefficient of $X^{k_{1}} Y^{k_{2}} Z^{k_{3}}$ in the development of $(X+Y+Z)^{n}$,

$$
(X+Y+Z)^{n}=\sum_{k_{1} \geq 0, k_{2} \geq 0, k_{3} \geq 0, k_{1}+k_{2}+k_{3}=n} \alpha_{k_{1} k_{2} k_{3}} X^{k_{1}} Y^{k_{2}} Z^{k_{3}},
$$

the Bézier polynomials of degree $n$ are

$$
B_{k_{1} k_{2} k_{3}}^{n}=\alpha_{k_{1} k_{2} k_{3}} \mathcal{N}_{A_{1}}^{k_{1}} \mathcal{N}_{A_{2}}^{k_{2}} \mathcal{N}_{A_{3}}^{k_{3}} .
$$

This polynomial is associated to the degree of freedom $\sigma$ defined by the barycentric coordinates $\mathcal{N}_{A_{l}}(\sigma)=\frac{k_{l}}{n}$. In the following, we drop the subscript $k_{1}, k_{2}, k_{3}$ and set instead $\sigma$ the point of $T$ that corresponds to $\left(k_{1}, k_{2}, k_{3}\right)$.

Clearly $B_{\sigma}^{n} \geq 0$ and $\sum_{\sigma \in T} B_{\sigma}^{n}=1$. The Bézier polynomials also have other properties such as a total variation diminishing property, see for example [19]. These two properties are in contrast with the Lagrange interpolant. Another difference is that the coefficients $u_{\sigma}$ in the development

$$
u_{\mid T}^{h}=\sum_{\sigma \in T} u_{\sigma} B_{\sigma}^{n}
$$


are not equal to $u^{h}(\sigma)$, contrarily to what happens for the Lagrange expansion of the same $u^{h}$,

$$
u_{\mid T}^{h}=\sum_{\sigma \in T} u^{h}(\sigma) L_{\sigma}^{n} .
$$

These properties are also shared by NURBS.

In the numerical examples, we have chosen $n=2$. The method presented above has been tested on two examples, a steady Burger-like equation and a problem with a non-convex flux.

\subsection{Example of the Burgers equation}

The first example is

$$
\begin{gathered}
\frac{\partial u}{\partial y}+\frac{1}{2} \frac{\partial u^{2}}{\partial x}=0 \quad \text { if } x \in[0,1]^{2} \\
u(x, y)=1.5-2 x \quad \text { on the boundary. }
\end{gathered}
$$

The exact solution consists in a fan that merges into a shock which foot is located at $(x, y)=(3 / 4,1 / 2)$. More precisely, the exact solution is

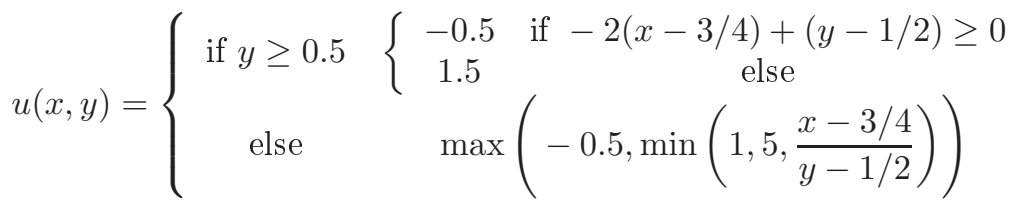

All the simulations are made using a regular mesh $M_{1}$ of 3192 vertices and 6192 triangles. In Figure 1, we show, for comparison purpose, the results of the second order scheme on the mesh $M_{2}$ where each triangle of $M_{1}$ is subdivided into four sub-triangles defined using the vertices and the mid point edges. We show the results with (21) and (22) and those where $\theta_{T}=0$.

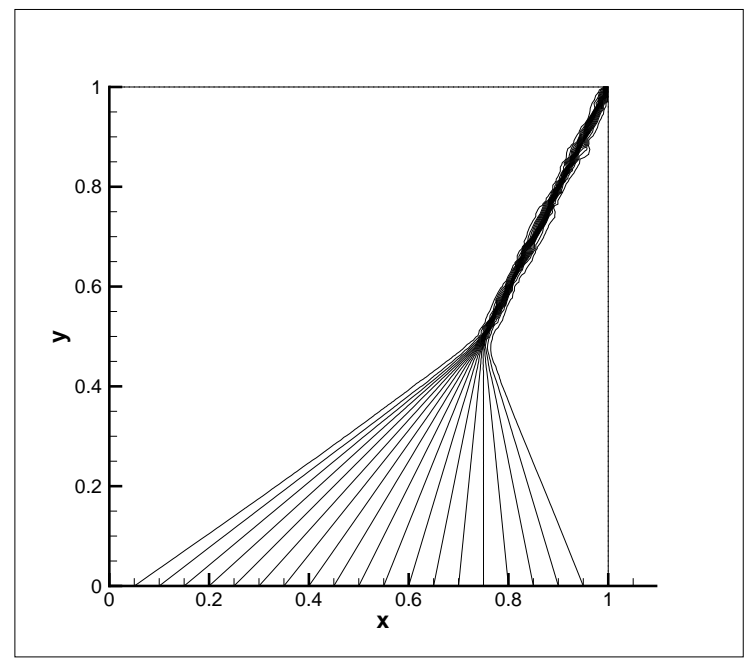

(a)

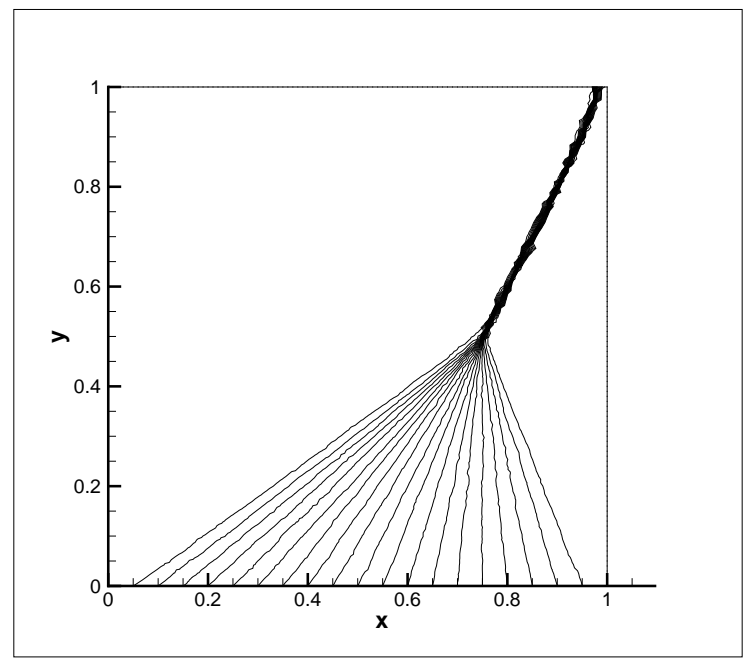

(b)

Figure 1: Second order solution of (23). The solution with the term (22) is (a), (b) is the solution without this term.

Then, we show the results obtained on $M_{1}$ using the Lagrange interpolation and the Bézier polynomials. The figure 2 show that there is almost no difference on the solutions (a) and (c). The solution (b) is more wiggly than (d). Let us emphasis that these wiggles are not any manifestation of instability. 


\subsection{Guckenheimer problem}

The method also works on more complex scalar examples such as the Guckenheim Riemann problem. Contrarily to the Burgers equation, the flux are no more convex, this modifies the structure of shocks and fans. We provide this example for two reasons : the structure are more complex, in particular a fan is ended by a shock. The second reason is that, since we do not have any mathematical analysis, it is interesting to see that, even for these non convex equations, it seems that the entropy condition is properly met.

The problem is to solve

$$
u(x, y, 0)=\left\{\begin{array}{cl}
0 & \frac{\partial u}{\partial t}+\frac{1}{2} \frac{\partial u^{2}}{\partial x}+\frac{1}{3} \frac{\partial u^{3}}{\partial y}=0 \\
1 & \text { if } 0<\arctan \left(\frac{y}{x}\right)<\frac{3 \pi}{4} \\
1 & \text { if } \frac{3 \pi}{2}<\arctan \left(\frac{y}{x}\right)<\frac{3 \pi}{2} \\
-1 & \text { irctan }\left(\frac{y}{x}\right)<2 \pi
\end{array}\right.
$$

The solution is self similar, $u(x, y, t)=v\left(\frac{x}{t}, \frac{y}{t}\right)$, and the function $v$ satisfies

$$
-\xi v_{\xi}-\nu v_{\nu}+\frac{1}{2} \frac{\partial u^{2}}{\partial \xi}+\frac{1}{3} \frac{\partial u^{3}}{\partial \nu}=0
$$

with the boundary conditions

$$
\lim _{r \rightarrow+\infty} v(r \cos \theta, r \sin \theta)=u(\cos \theta, \sin \theta, 0) .
$$

Solving (25) amounts to solve (24) at $t=1$.

This problem has been discussed in [21] and drawn to our attention by M. Ben Artzi (Hebrew University of Jerusalem). The flux $g(u)=\frac{u^{3}}{3}$ is non convex and this induces sonic shocks. The exact solution consists in

- A shock coming out from the line $y=0$ that moves at the speed $1 / 3$ in the positive direction,

- a steady shock at $x=0$,

- A shock coming out from the line $x+y=0$. The analysis of [21] by a self similar analysis indicates that the location of this shock is $x+y-5 / 6 t$, with in our case, $t=1$.

From the numerics, (25a) is rewritten as

$$
\frac{\partial F(u)}{\partial \xi}+\frac{\partial G(u)}{\partial \nu}+2 u=0
$$

with $F(u)=\frac{1}{2} u^{2}-\xi u$ and $G(u)=\frac{1}{3} u^{3}-\nu u$. The total residual on $T$ writes

$$
\Phi^{T}=\int_{\partial T}\left(F(u) n_{x}+G(u) n_{y}\right) d x d y+2 \int_{T} u d x d y
$$

that are evaluated by numerical quadratures. The integral on $\partial T$ uses 3 point Gaussian quadrature formula. The integral on $T$ use the weights and points (in barycentric coordinates) of table 2. This quadrature formula, taken from [22], page 184, is 4th order accurate (exact for cubic polynomials).

The solution is displayed on figures 3 and 4 . We see that even for this non convex problem, there is no stability problem. Again, we notice that the Bézier solution, without the the additional term (22) is less wiggly that the one obtained with Lagrange interpolant. The other interesting observation is that the correct entropy solution is recovered in that case, as in all the other cases of this paper, without any additional feature, even without the additional stabilization. 


\begin{tabular}{||ccc||}
\hline multiplicity & coordinates & weight \\
\hline 3 & $\left(x_{0}, x_{0}, 1-2 x_{0}\right)$ & $\omega_{0}$ \\
3 & $\left(x_{1}, x_{1}, 1-2 x_{1}\right)$ & $\omega_{1}$ \\
\hline
\end{tabular}

Table 2: Quadrature points and weights. The other points are obtained by cyclic permutation of the barycentric coordinates. We have set $x_{0}=0.445948490915965, \omega=0.223381589678010, x_{1}=0.091576213509771$, $\omega_{1}=0.109951743655322$.

\section{Extension to the wave equation}

The case of the wave equation can also be set up in a similar way. The wave equation writes

$$
\begin{array}{ll}
\frac{\partial U}{\partial t}+A \frac{\partial U}{\partial x}+B \frac{\partial U}{\partial y}=0 & \text { in } \Omega \\
K_{\vec{n}}^{+}(U-g)=0 & \vec{n} \text { outward unit normal at } \partial \Omega
\end{array}
$$

with $U=\left(u, v_{1}, v_{2}\right)$,

$$
A=c\left(\begin{array}{lll}
0 & 1 & 0 \\
1 & 0 & 0 \\
0 & 0 & 0
\end{array}\right) \text { and } B=c\left(\begin{array}{lll}
0 & 0 & 1 \\
0 & 0 & 0 \\
1 & 0 & 0
\end{array}\right)
$$

If $\vec{n}=\left(n_{x}, n_{y}\right)$, the matrix $K_{\vec{n}}=n_{x} A+n_{y} B$ admits three eigenvalues, namely $\lambda=0, c\|n\|,-c\|n\|$. It is well known that the system is hyperbolic.

In order to discretize the system (27) using Residual distribution schemes, it is not possible to rely on the method of lines. The key reason for that is that the accuracy of the method: as explained in proposition 1.2 the structure of the equation must be plugged into the scheme. Hence, we need to preserve the coupling between the time and space operators.

In order to overcome this difficulty, one solution is first to discretize in time and then to see the semidiscrete problem as a steady one with a source term.

In the examples, we approximate $(27)$ as

$$
\frac{3}{2} \frac{U^{n+1}-U^{n}}{\Delta t}-\frac{1}{2} \frac{U^{n}-U^{n-1}}{\Delta t}+A{\frac{\partial U^{n+1}}{\partial x}}^{n}+B{\frac{\partial U^{n+1}}{\partial y}}^{n}=0
$$

which is seen as

$$
\alpha U^{n+1}+A{\frac{\partial U^{n+1}}{\partial x}}^{n+B} \frac{\partial U}{\partial y}^{n+1}+S=0
$$

with

$$
S=-\frac{3}{2} \frac{U^{n}}{\Delta t}-\frac{1}{2} \frac{U^{n}-U^{n-1}}{\Delta t}
$$

In the specific example we consider for the numerics, we take $g=0$ and

$$
K_{n}^{+} U^{n+1}=0
$$

\subsection{Scheme description}

We extend the LDA scheme described for steady problems in [13]. We use Bézier polynomials because $B_{\sigma}^{2} \geq 0$, hence, for any of the sub triangles of figure 5

$$
\int_{T_{\xi}}\left(\frac{3}{2} \frac{U^{n+1}-U^{n}}{\Delta t}-\frac{1}{2} \frac{U^{n}-U^{n-1}}{\Delta t}\right) d x=\sum_{\sigma \in T} \omega_{\sigma}^{T_{\xi}}\left(\frac{3}{2} \frac{U_{\sigma}^{n+1}-U_{\sigma}^{n}}{\Delta t}-\frac{1}{2} \frac{U_{\sigma}^{n}-U_{\sigma}^{n-1}}{\Delta t}\right)
$$


with $\omega_{\sigma}^{T_{\xi}}>0$. This ensure that each degree of freedom will have a contribution in the scheme. This would have been wrong for Lagrange interpolation since the integral over $T$ of the basis functions at the vertices of $T$ is zero. Indeed, a simple calculation shows that a similar formula can be obtained, but here we would have negative weights.

We define $\vec{n}_{\sigma}^{T_{\xi}}$ as the scaled inwards normal vector to the edge of $T_{\xi}, \xi=I, I I, I I I, I V$, opposite to $\sigma \in T_{\xi}$. For each sub-triangle, we define the sub-residuals,

$$
\Phi^{T_{\xi}}=\int_{T_{\xi}}\left(\frac{3}{2} \frac{U^{n+1}-U^{n}}{\Delta t}-\frac{1}{2} \frac{U^{n}-U^{n-1}}{\Delta t}+A \frac{\partial U^{n+1}}{\partial x}+B \frac{\partial U^{n+1}}{\partial y}\right) d x
$$

and the split-residuals for $\sigma \in T_{\xi}$

$$
\Phi_{\sigma}^{T_{\xi}}=K_{\vec{n}_{\sigma}^{T \xi}}^{+} N_{T_{\xi}} \Phi^{T_{\xi}}
$$

with

$$
N_{T_{\xi}}=\left(\sum_{\sigma \in T_{\xi}} K_{\vec{n}_{\sigma}^{T \xi}}^{+}\right)^{-1}
$$

Using the arguments of [10], we can easily see that $\sum_{\sigma \in T_{\xi}} K_{\vec{n}_{\sigma}^{T}}^{+}$is invertible because the matrices $A$ and $B$ do not commute. The last step of the scheme definition is

$$
\sum_{\xi: T_{\xi} \ni \sigma} \Phi_{\sigma}^{T_{\xi}}=0
$$

The scheme (32) with the boundary conditions (28b) with (29), (30), (31) is implicit in $U^{n+1}$. In order to compute it, we use an iterative technique similar to what is done for the steady problems, this defines a sequence $U^{n+1, k}$ with $k \in \mathbb{N}$. We take $U^{n+1,0}=U^{n}$. The boundary condition (28b) is applied at each iteration: $K_{\vec{n}}^{+} U^{n+1, k}=0$. The iterative process is stopped once a given threshold (relative error of $10^{-3}$ ) for some integer $k=k_{\max }$ is reached and we set $U^{n+1}=U^{n+1, k_{\max }}$

\subsection{Some numerical results}

We have used a regular mesh of $80 \times 80$ quadrangles, each triangle is cut into two triangles. The domain $\Omega$ is $[-4,4] \times[-4,4]$ and the speed of sound is set to $c=1$.

We have plotted the $u$ component of the solution. It is displayed in the figures $6,7,8$. The results are very regular and compare well to those obtained by M. Duruflé (U. Bordeaux I) with his code Montjoie ${ }^{1}$.

\section{Concluding remarks}

We have developed a general method that enables to compute steady and unsteady solutions of linear and non linear wave problems. It relies on a approximation technique that can use either Lagrange or non Lagrange approximation function. An example with Bézier polynomial has been given. The improvement with respect to previous Residual distribution schemes is that the degrees of freedom are no longer interpreted as point values. This remark opens the way to more general approximation methods such as the isogeometric analysis as what is currently being developed by Hughes and coworkers with schemes that has non oscillatory properties without tuning parameters.

\footnotetext{
${ }^{1}$ http://www.math.u-bordeaux1.fr/ durufle/montjoie/index.php
} 


\section{Acknowledgements}

RA has been financed in part by the EU Strep ADIGMA and the FP7 Idea Advanced Grant "ADDECCO" No 226316. JK has been financed by the AEROCAV grant of the INCA program of SNECMA. Marc Duruflé (Institut Polytechnique de Bordeaux, Institut de Mathématiques de Bordeaux) is warmly thanks enabling us to check our results against his DG code.

\section{References}

[1] P. L. Roe. Approximate Riemann solvers, parameter vectors, and difference schemes. J. Comput. Phys., 43, 1983.

[2] R.-H. Ni. A multiple grid scheme for solving the Euler equations. AIAA J., 20(11):1565-1571, 1981.

[3] T.J.R. Hughes, L.P. Franca, and M. Mallet. A new finite element formulation for CFD: I. symmetric forms of the compressible Euler and Navier-Stokes equations and the second law of thermodynamics. Comp. Meth. Appl. Mech. Engrg., 54:223-234, 1986.

[4] T.J.R. Hughes and M. Mallet. A new finite element formulation for CFD: III. the generalized streamline operator for multidimensional advective-diffusive systems. Comp. Meth. Appl. Mech. Engrg., 58:305$328,1986$.

[5] C. Johnson. In Numerical Solution of Partial Differential Equations by the Finite Element Method. Cambridge University Press, Cambridge, 1987.

[6] R. Struijs, H. Deconinck, and P.L. Roe. Fluctuation splitting schemes for the 2D Euler equations. VKI-LS 1991-01, 1991. Computational Fluid Dynamics.

[7] H. Paillère, J.-C. Carette, and H. Deconinck. Multidimentional upwind and supg methods for the solution of the compressible flow equations on unstructured grids. VKI-LS 1994-05, 1994. Computational Fluid Dynamics.

[8] E. van der Weide, H. Deconinck, E. Issmann, and G. Degrez. Fluctuation splitting schemes for multidimensional convection problems: an alternative to finite volume and finite element methods. Сomputational Mechanics, 23(2):199-208, 1999.

[9] Á. Csík, M. Ricchiuto, and H. Deconinck. A conservative formulation of the multidimensional upwind residual distribution schemes for general nonlinear conservation laws. J. Comput. Phys, 179(2):286-312, 2002.

[10] R. Abgrall. Toward the ultimate conservative scheme: Following the quest. J. Comput. Phys., 167(2):277-315, 2001

[11] R. Abgrall and M. Mezine. Construction of second-order accurate monotone and stable residual distribution schemes for steady problems. J. Comput. Phys., 195(2):474-507, 2004.

[12] R. Abgrall and M. Mezine. Construction of second order accurate monotone and stable residual distribution schemes for unsteady flow problems. J. Comput. Phys., 188(1):16-55, 2003.

[13] R. Abgrall and L. Roe, P. High-order fluctuation schemes on triangular meshes. J. Sci. Comput., 19(1-3):3-36, 2003.

[14] R. Abgrall and C.W. Shu. Development of residual distribution schemes for the discontinuous galerkin methods : the scalar case. Communication in Computational Physics, 9:376-390, 2009. 
[15] M.E. Hubbard. A framework for discontinuous fluctuation distribution. Int. J. Numer. Methods Fluids, 56(8):1305-1311, 2008.

[16] T.J.R. Hughes, J.A. Cottrell, and Y. Bazilevs. Isogeometric analysis: CAD, finite elements, NURBS, exact geometry and mesh refinement. Comput. Methods Appl. Mech. Eng., 194(39-41):4135-4195, 2005.

[17] P.G. Ciarlet and P.A. Raviart. General Lagrange and Hermite interpolation in $R^{n}$ with applications to finite element methods. Arch. Ration. Mech. Anal., 46:177-199, 1972.

[18] C. de Boor. A practical guide to splines. Number 27 in Applied mathematical sciences. Springer, 1978.

[19] David F. Rogers. An introduction to NURBS : with historical perspectives. Morgan Kaufman, 2001.

[20] R. Abgrall, M. Ricchiutto, and A. Larat. A simple construction of very high order non oscillatory compact schemes on unstructured meshes. Computers and Fluids, 2008. in press.

[21] J. Guckenheimer. Shocks and rarefactions in two space dimensions. Arch. Ration. Mech. Anal., 59:281$291,1975$.

[22] G. Strang and G.J. Fix. An Analysis of the Finite Element Method. Prentice-Hall, Englewood Cliffs, N.J., 1973. 


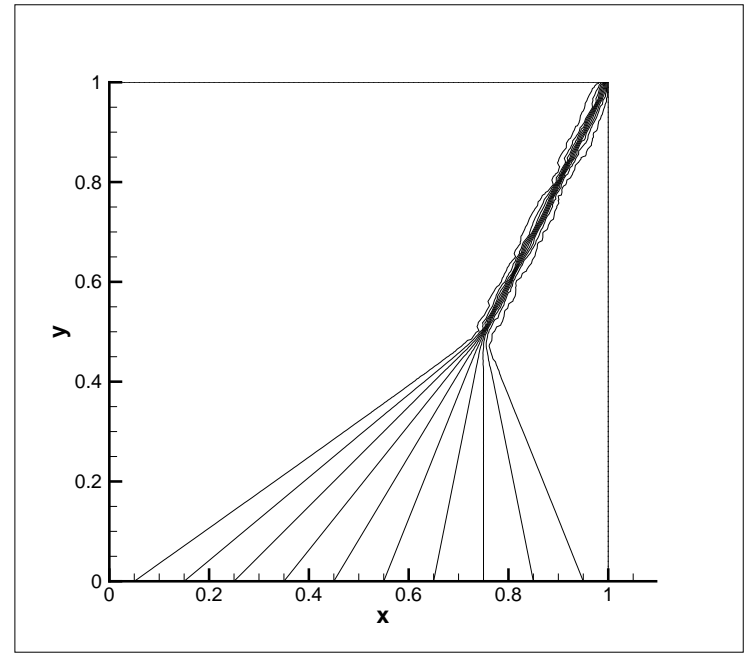

(a)

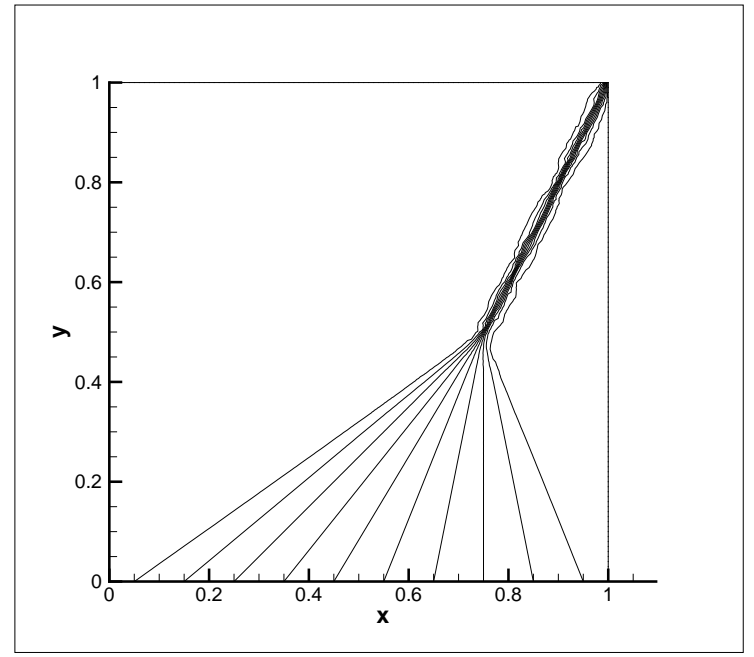

(c)

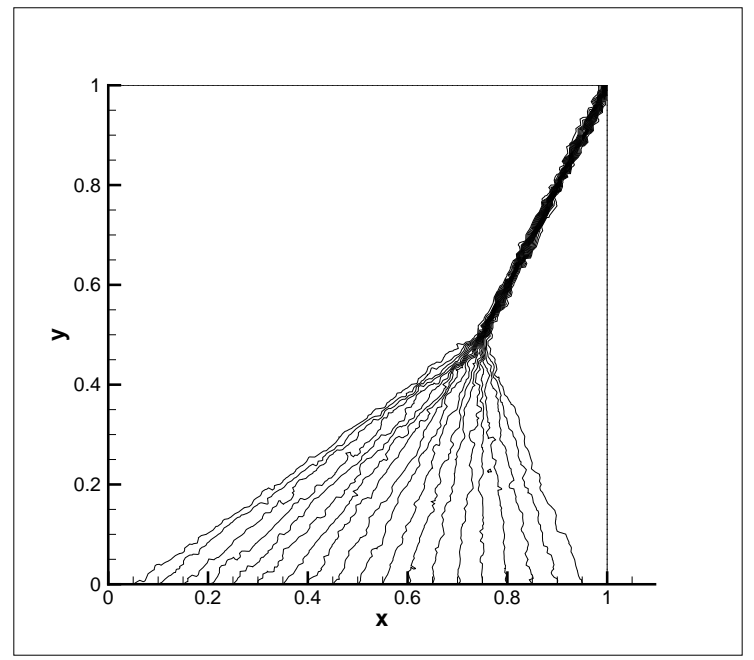

(b)

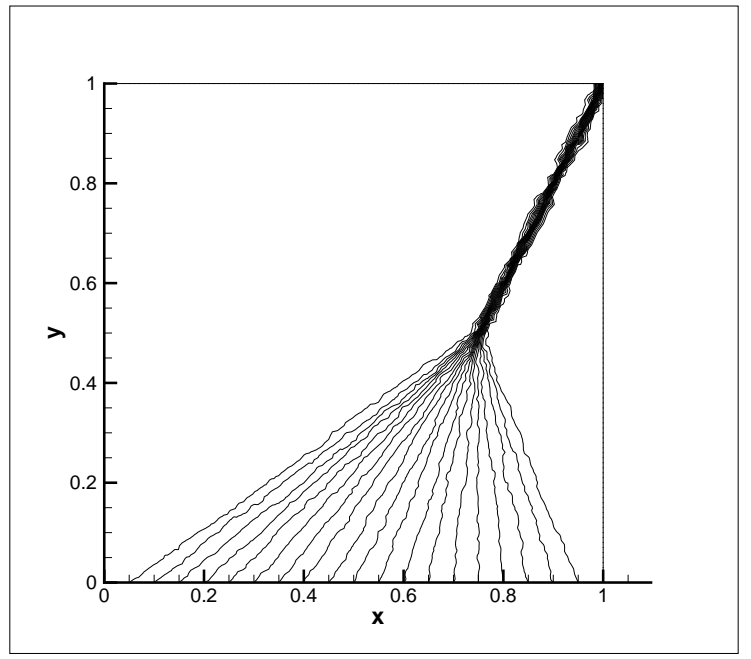

(d)

Figure 2: Third order solution of (23). The solution with the term (22) are (a) and (c), (b) and (d) are the solutions without this term. The solutions (a) and (b) are obtained with the Lagrange interpolant and (c) and (d) are obtained by the Bézier ones. 


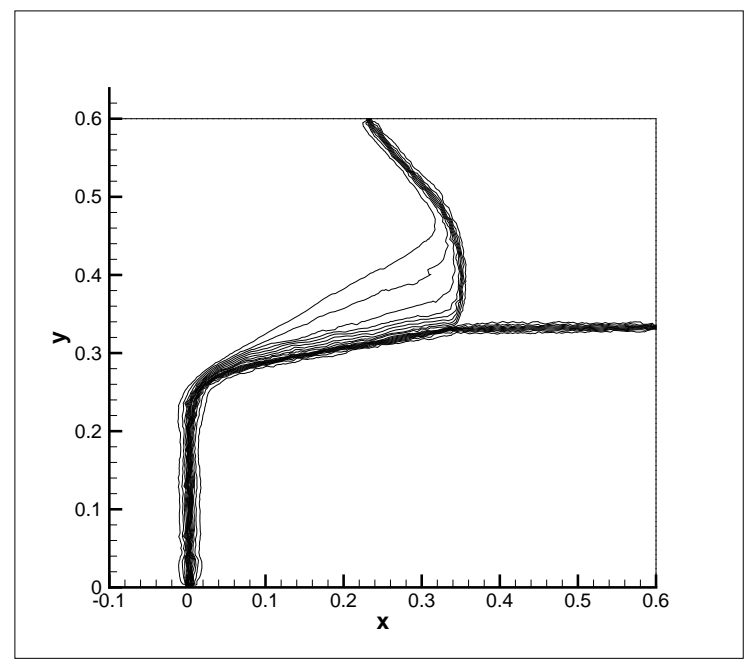

(a)

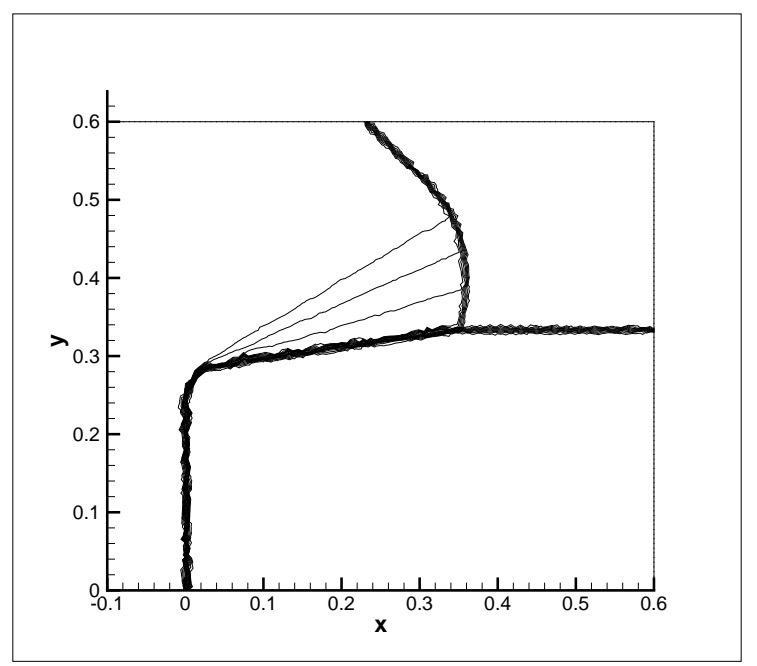

(b)

Figure 3: Second order solution of (25). The solution with the term (22) is (a), (b) is the solution without this term. 


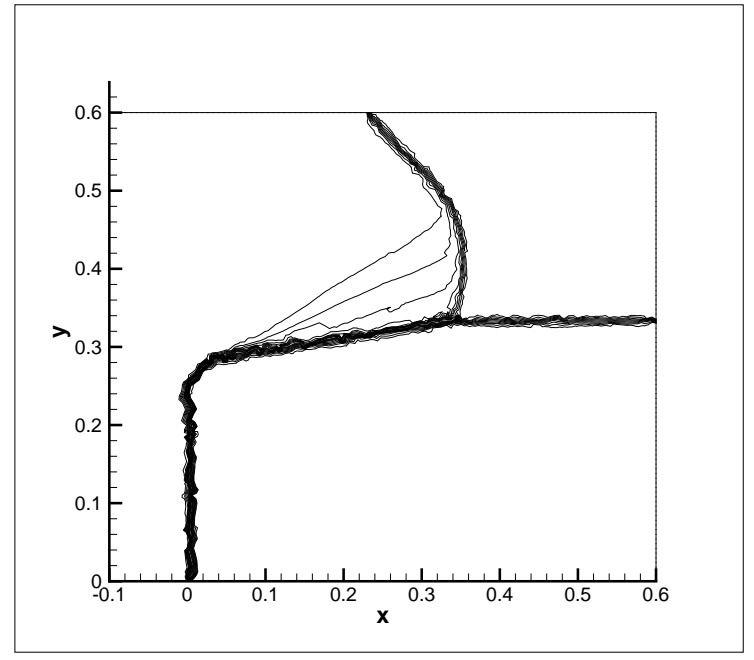

(a)

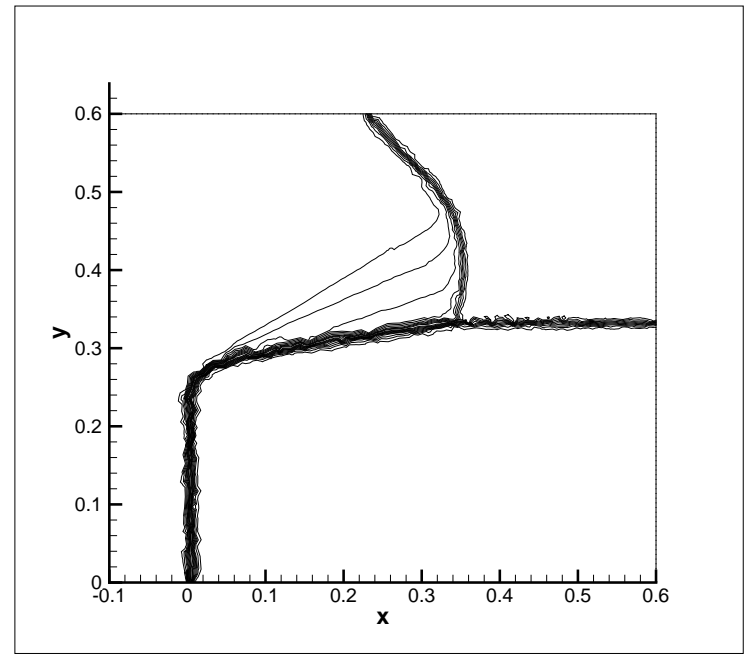

(c)

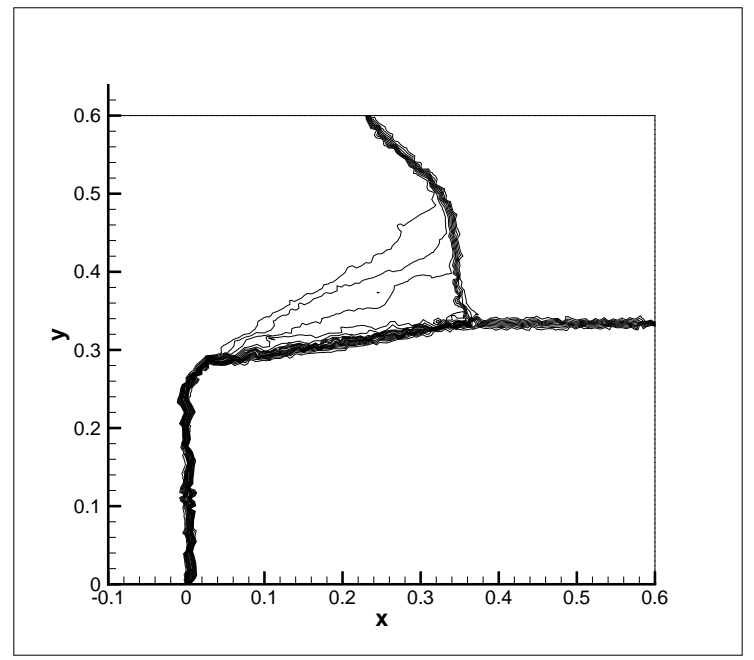

(b)

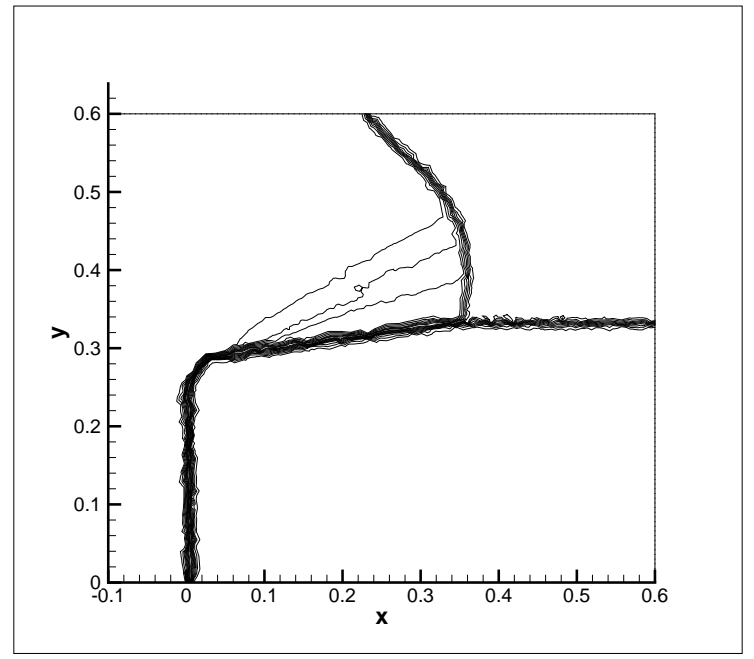

(d)

Figure 4: Third order solution of (25). The solution with the term (22) are (a) and (c), (b) and (d) are the solutions without this term. The solutions (a) and (b) are obtained with the Lagrange interpolant and (c) and (d) are obtained by the Bézier ones. 


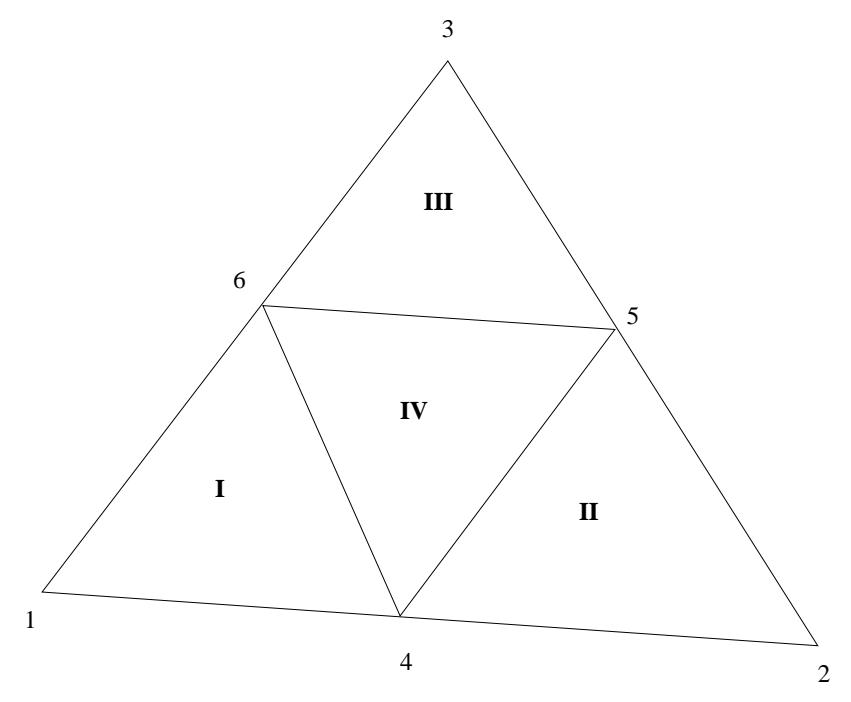

Figure 5: Definitions of the sub-triangles in $T$.
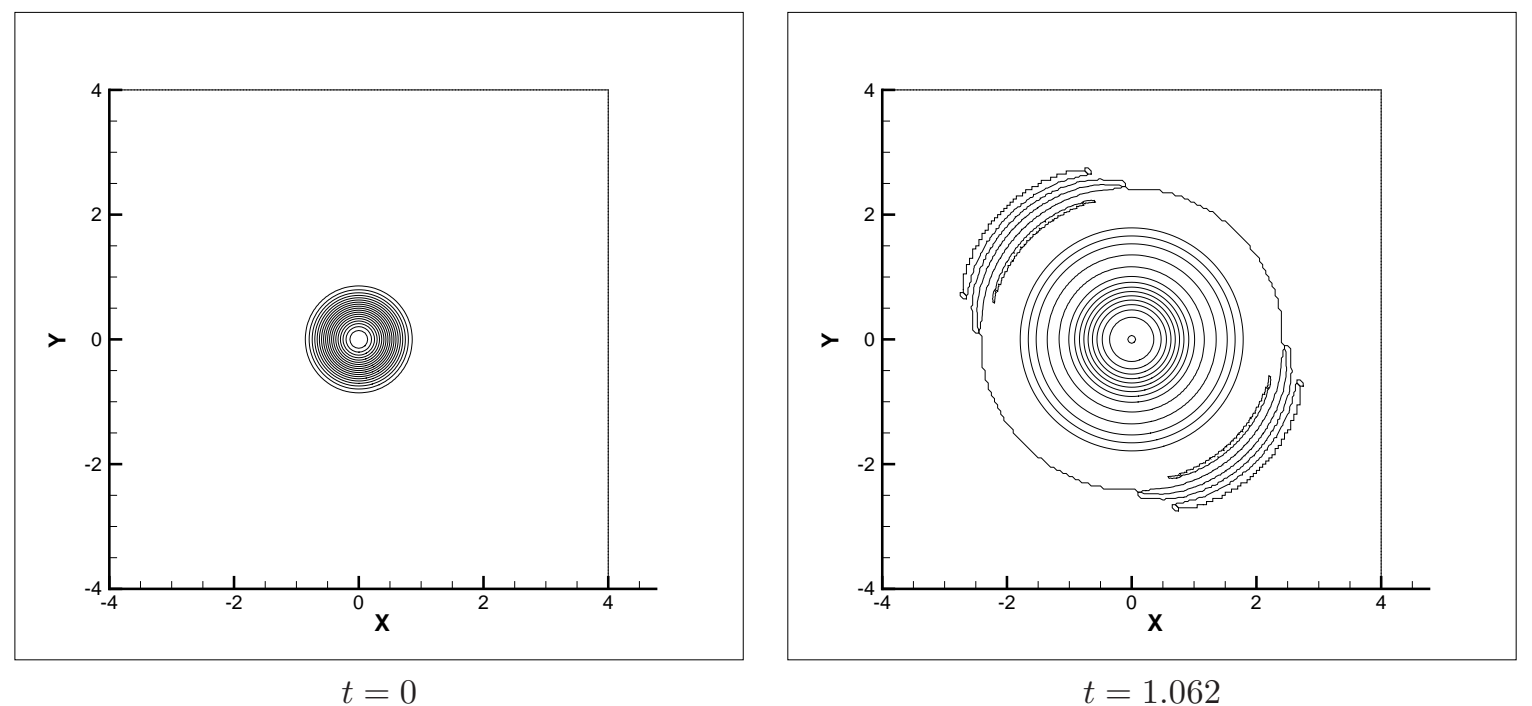

Figure 6: Two instances of the solution. In each case, 15 isolines of the solution is displayed : the scale is different from one figure to the other. 


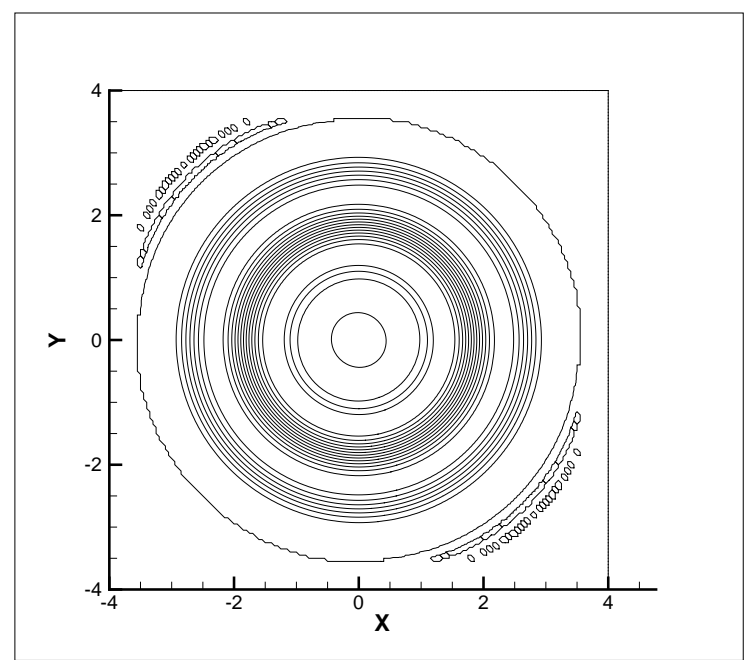

$t=2.123$

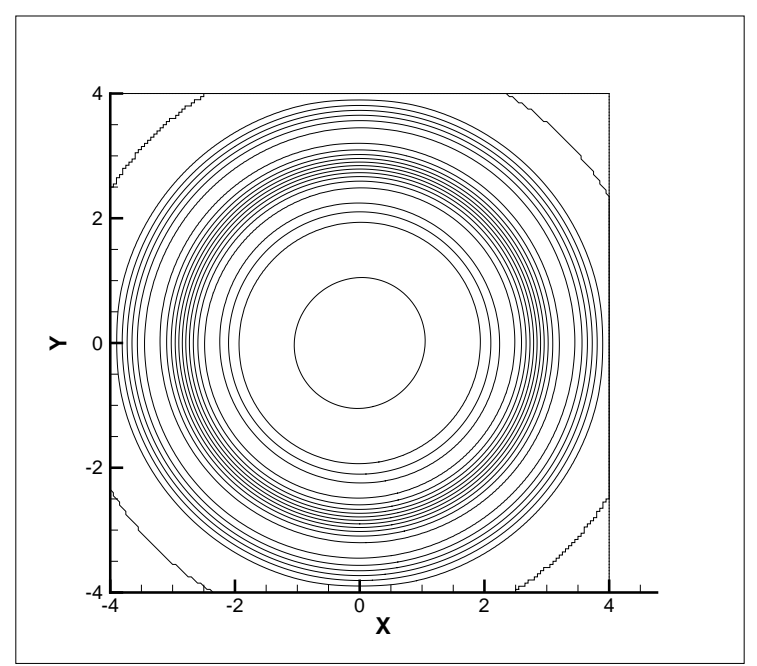

$t=3.185$

Figure 7: Two instances of the solution. In each case, 15 isolines of the solution is displayed : the scale is different from one figure to the other.

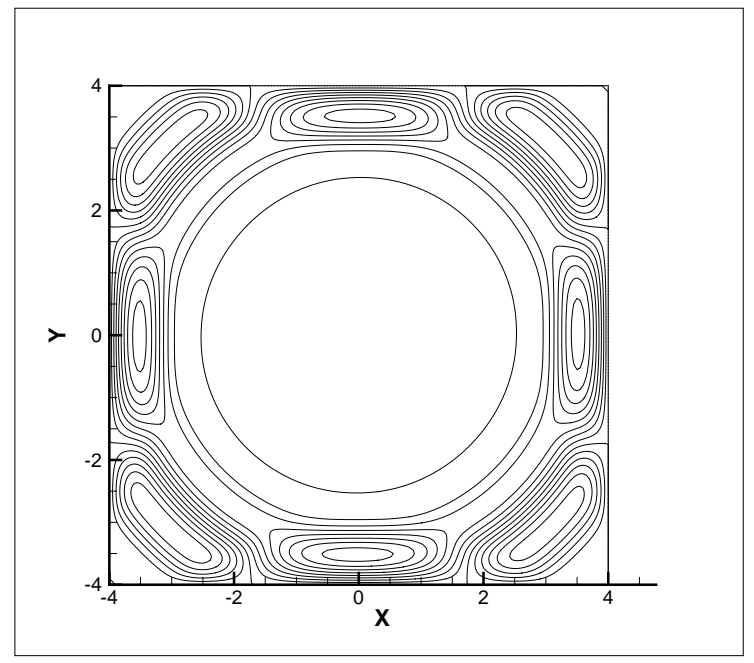

$t=4.246$

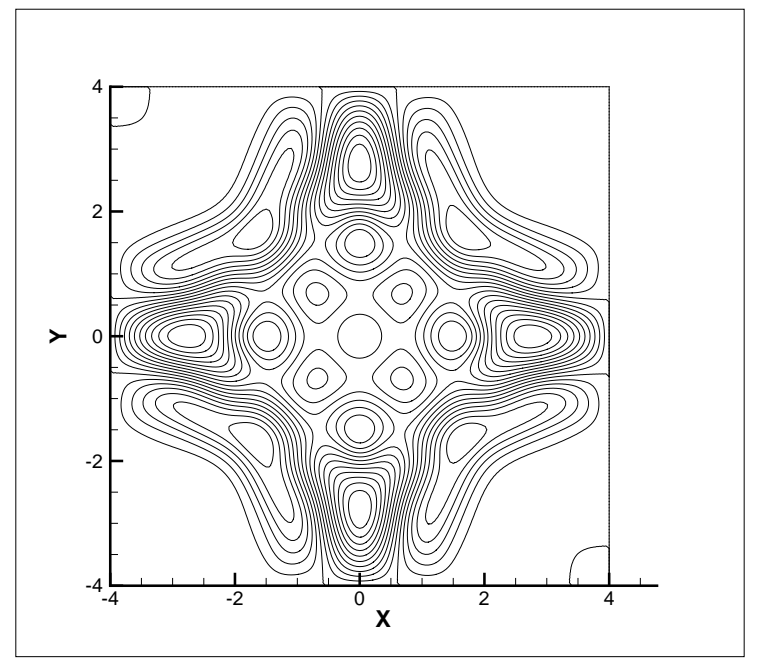

$t=9.554$

Figure 8: Several instance of the solution. In each case, 15 isolines of the solution is displayed : the scale is different from one figure to the other. 